\title{
Correction to: MLASP: Machine learning assisted capacity planning. An industrial experience report
}

\author{
Arthur Vitui $^{1,2}$ (D) $\cdot$ Tse-Hsun (Peter) Chen ${ }^{1}$
}

Published online: 16 August 2021

๑ Springer Science+Business Media, LLC, part of Springer Nature 2021

\section{Correction to: Empirical Software Engineering https://doi.org/10.1007/s10664-021-09994-0}

The original version of this article unfortunately contained mistakes.

(1) The second affiliation of the author, Arthur Vitui, was incorrect. The correct information is given below.

(2) The image captured for Figure 6 was incorrect. The correct version is given below.

The original article has been corrected.

The original article can be found online at https://doi.org/10.1007/s10664-021-09994-0.

\section{Arthur Vitui}

arthur.vitui@mail.concordia.ca

Tse-Hsun (Peter) Chen

peterc@encs.concordia.ca

1 Software PErformance, Analysis, and Reliability (SPEAR) Lab, Concordia University, Montreal, Canada

2 Red Hat Inc, Toronto, Canada 

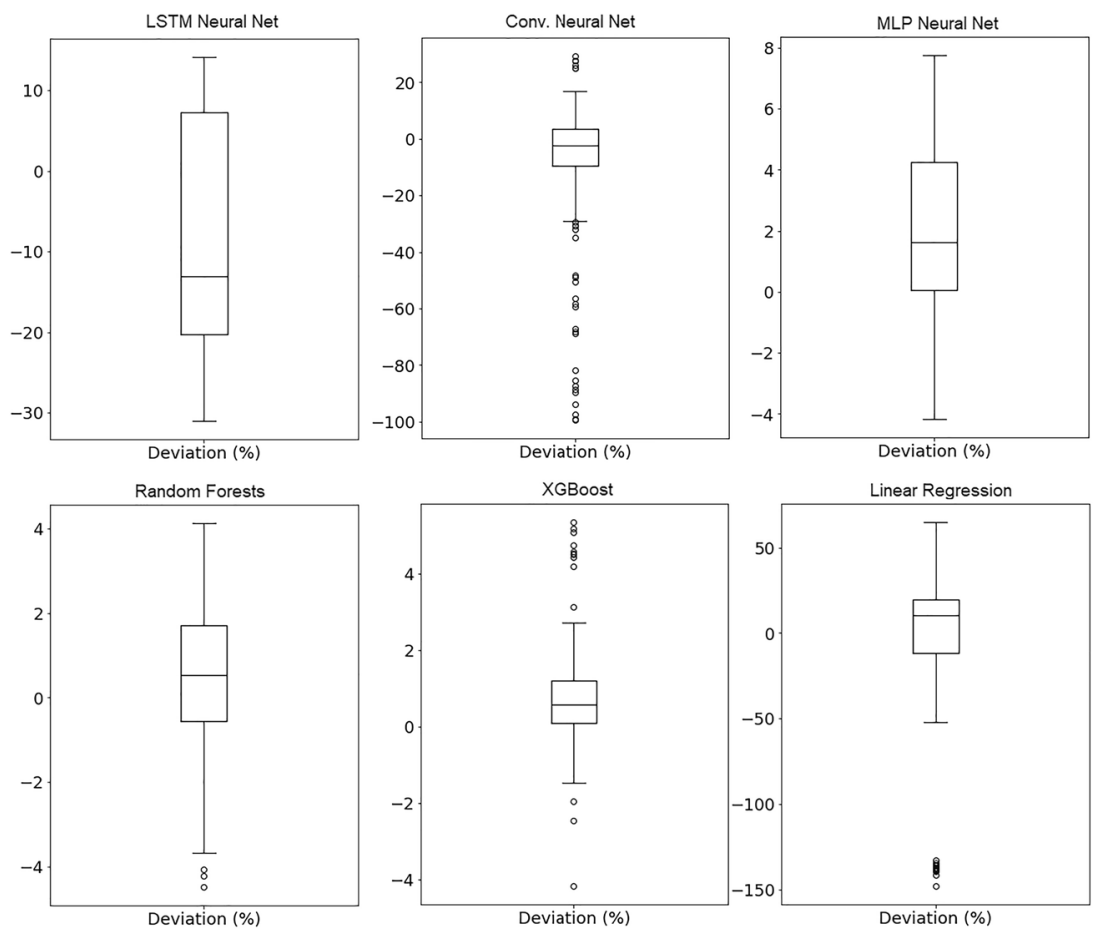

Fig. 6 Distributions of the percentage deviation between the predicted and actual value for the open-source system using a subset of data to train the models

Publisher's note Springer Nature remains neutral with regard to jurisdictional claims in published maps and institutional affiliations. 